\section{CLINICAL REPORT}

\section{G. Zuccoli}

A. Panigrahy

A. Bailey

C. Fitz

\title{
Redefining the Guillain-Barré Spectrum in Children: Neuroimaging Findings of Cranial Nerve Involvement
}

\begin{abstract}
SUMMARY: GBS and its MFS variant are acute polyneuropathies that are considered to represent a continuum rather than distinct entities, due to the overlap in their clinical features. Enhancement of the CE roots represents the neuroradiologic hallmark of GBS, while findings of neuroimaging studies in MFS are usually unremarkable. Our purpose was to evaluate the MR imaging findings of polyneuropathy in 17 children affected by GBS and its MFS variant. Fourteen of our 17 patients demonstrated CE enhancement, with predominant involvement of the anterior roots. Of 6 patients who underwent MR imaging of the brain, 5 had cranial nerve involvement. In children affected by GBS-MFS, involvement of the CE roots may be considered part of a more extensive autoimmune neuropathy, as demonstrated by enhancement of cranial nerves. Brain MR imaging should be considered in the routine evaluation in pediatric patients with GBS-MFS for the evaluation of the cranial nerves.
\end{abstract}

ABBREVIATIONS: $\mathrm{CE}=$ cauda equina; GBS = Guillain-Barré Syndrome; MFS = Miller Fisher syndrome

G BS is a rapidly progressing peripheral inflammatory polyneuropathy that causes acute neuromuscular failure. The incidence of GBS has been reported to be $1-2$ per 100,000 in the general population. ${ }^{1-3}$ A mild respiratory or gastrointestinal tract infection precedes onset of symptoms in 75\% of patients with GBS. ${ }^{4,5}$ It has been suggested that surgical procedures, lymphoma, and systemic lupus erythematosus may also be predisposing conditions for GBS. ${ }^{4}$

Presentation of GBS is initially characterized by progressive and ascending weakness of the extremities and areflexia. Progression of symptoms is rapid and may include sensory disturbances, facial nerve palsies, autonomic dysfunction, pain, numbness, paraesthesia, and respiratory failure. ${ }^{6,7}$ Diagnosis is usually established on the basis of the clinical presentation but may be aided by CSF characteristics, electrophysiologic examination, and neuroimaging findings. An elevated CSF protein level without pleocytosis is usually indicative of GBS. $^{7,8}$

Many symptoms of GBS are nonspecific, and because the diagnosis is based mainly on clinical features, MR imaging is an important tool used in the differential diagnosis.

MFS is considered the cranial nerve variant of GBS, and diagnosis is based on the triad of ophthalmoplegia, ataxia, and areflexia. $^{9,10}$ In GBS, neuroimaging demonstrates involvement of the CE roots; however, in MFS, MR imaging findings are usually within normal limits. ${ }^{11}$ The aim of this study was to review the MR imaging findings of polyneuropathy in children affected by either GBS or the MFS variant and to assess

Received August 14, 2010; accepted after revision September 6.

From the Department of Radiology (G.Z., A.P., A.B., C.F.), University of Pittsburgh Medical Center, Children's Hospital of Pittsburgh, Pittsburgh, Pennsylvania.

Please address correspondence to: Giulio Zuccoli, MD, Department of Radiology, University of Pittsburgh Medical Center, Children's Hospital of Pittsburgh, PA 15224; e-mail:giulio. zuccoli@gmail.com

Indicates open access to non-subscribers at www.ajnr.org

DOI 10.3174/ajnr.A2358 whether these diagnoses constitute a clinical-neuroradiologic spectrum.

\section{Materials and Methods}

Our study group included 17 pediatric patients with GBS who were retrospectively selected from the patients seen at our institution between 2001 and 2010. The clinical diagnostic criteria for GBS proposed by van Doorn et al in $2008^{7}$ were used in this study. The 10 criteria required for and highly suggestive of GBS include progressive weakness in both arms and legs (it might start with weakness only in the legs); areflexia (or decreased tendon reflexes); progression of symptoms for days to 4 weeks; relative symmetry of symptoms; mild sensory symptoms or signs; cranial nerve involvement, especially bilateral weakness of facial muscles; autonomic dysfunction; pain; and a high concentration of protein in the CSF. ${ }^{7}$ The triad of ophthalmoplegia, ataxia, and areflexia proposed by Fisher in $1956^{9}$ required for the diagnosis of MFS was used in this study.

In our study group, GBS affected males more than females (59\% over $41 \%$, respectively). The mean age of our patient population was 9.41 years (range, 16 months to 18 years). Sixteen patients (94\%) experienced either a viral or respiratory infection within 3 weeks before the onset of symptoms. Our patient population demonstrated considerable variability in recovery time, from 2 weeks to 3 years. All patients were treated with intravenous immunoglobulinin therapy along with other treatment methods, including pain management (13 patients, $76.5 \%$ ), physical therapy (12 patients, $70.6 \%$ ), antibiotics (6 patients, $35.3 \%$ ), steroids (3 patients, $17.6 \%$ ), dietary supplementation (3 patients, $17.6 \%$ ), and respiratory support (3 patients, $17.6 \%$ ).

A lumbar puncture was performed in all patients, and the CSF was analyzed for increased protein levels $(>45 \mathrm{mg} / \mathrm{dL})$. Seven patients also underwent electromyography. All patients received full spinal MR imaging, and 6 patients also underwent cranial MR imaging. Patients were scanned with a $1.5 \mathrm{~T}$ magnet (Signa; GE Healthcare, Milwaukee, Wisconsin) by using a standard spinal and brain protocol that included short- and long-TR precontrast sequences. After the injection of $0.1 \mathrm{~mL} / \mathrm{kg}$ of gadolinium-based contrast material, $3-\mathrm{mm}$ sagittal and axial images were obtained through the spine. For those patients undergoing brain MR imaging, postcontrast images included 5-mm axial and coronal T1-weighted images. The images were retro- 


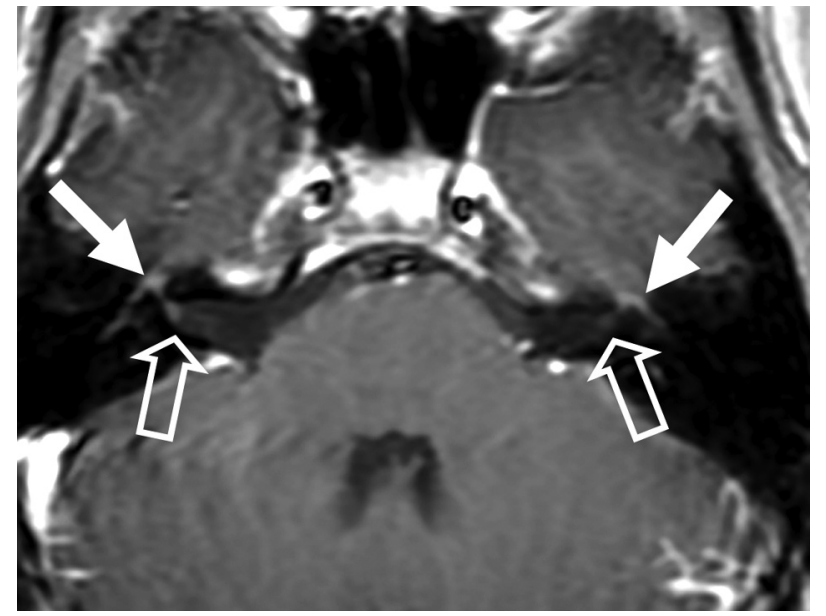

Fig 1. Patient 3. Bilateral enhancement of the intratemporal seventh cranial nerve is seen (solid arrows). Note the fundal enhancement of the seventh cranial nerve (open arrows), right greater than left.

spectively evaluated by 2 experienced pediatric neuroradiologists. The degree of enhancement of the CE roots was defined as absent, present, or strongly present. The anterior and posterior intrathecal roots were evaluated separately. Enhancement of the cranial nerves was defined as absent or present. Consensus was reached on discordant interpretations by a third pediatric neuroradiologist, independently. Brain images were evaluated for abnormal parenchymal signal-intensity alterations on all pulse sequences and pathologic enhancement of the cranial nerves.

\section{Results}

\section{Clinical and Laboratory Findings}

In our patient population, all patients experienced neuropathy and varying degrees of ataxia. Additional symptoms included areflexia (16 patients, 94.1\%), progressive weakness (16 patients, $94.1 \%$ ), pain (13 patients, $76.5 \%$ ), relative symmetry of symptoms ( 12 patients, $70.6 \%$ ), autonomic dysfunction ( 9 patients, $52.9 \%$ ), sensory symptoms (7 patients, $41.2 \%$ ), facial nerve palsy ( 5 patients, $29.4 \%$ ), ophthalmoplegia (4 patients, $23.5 \%$ ), and dysphagia (3 patients, $17.6 \%$ ). Twelve $(70.6 \%)$ patients were found to have elevated CSF protein levels. Results of extensive investigations for infectious, hematologic, and neoplastic disorders were negative. Of the 7 patients who underwent electrophysiologic studies, $6(85.7 \%)$ studies were positive for GBS. All of our patients, with the exception of 1 , demonstrated more than half $(>5)$ of the clinical features required for and highly suggestive of GBS. ${ }^{7}$ Most interesting, all of our patients also displayed at least 2 of the hallmark characteristics for the MFS variant of GBS, ${ }^{7,11}$ and 3 patients demonstrated the full triad.

\section{Neuroimaging Findings}

Involvement of the CE roots was observed in 14 patients $(82.3 \%)$. There was simultaneous enhancement of the anterior and posterior CE roots in 11 patients $(64.7 \%)$. Isolated enhancement of the anterior CE roots was observed in 3 patients $(17.6 \%)$. There was no evidence of isolated posterior CE root enhancement. Strong enhancement of the anterior CE roots was demonstrated in 9 patients $(52.9 \%)$, and 3 patients $(17.6 \%)$ had strong enhancement of the posterior roots. Most

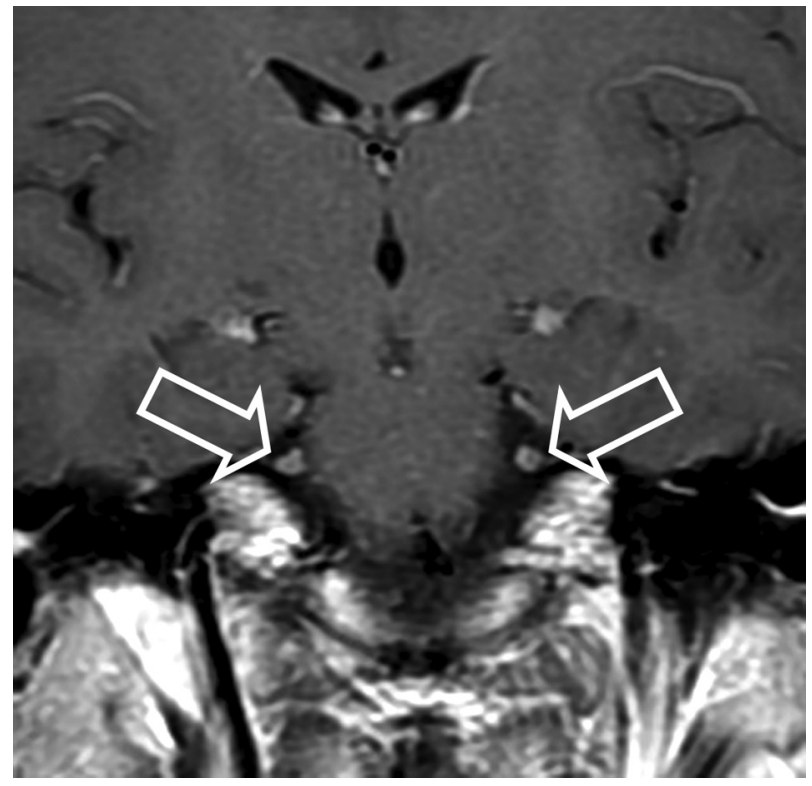

Fig 2. Patient 4. Bilateral enhancement of the fifth cranial nerve is observed on a coronal T1-weighted image (open arrows).

interesting, all 3 patients with strong enhancement of the posterior CE roots also had strong enhancement of the anterior roots. Of the 3 patients with the full MFS triad, 1 showed CE root enhancement.

Of 6 patients who had MR imaging of the brain, 5 patients (83.3\%) demonstrated cranial nerve involvement. The third and the sixth cranial nerves were the most commonly affected, but the fifth, seventh, eleventh, and twelfth cranial nerves also showed involvement. This involvement was associated with CE root enhancement in 1 of 3 patients who had the classic MFS triad. There was no evidence of abnormal brain parenchymal signal intensity on any of the pulse sequences examined.

\section{Discussion}

Our study demonstrates a high incidence of cranial nerve abnormalities in pediatric GBS and its MFS variant. These find-

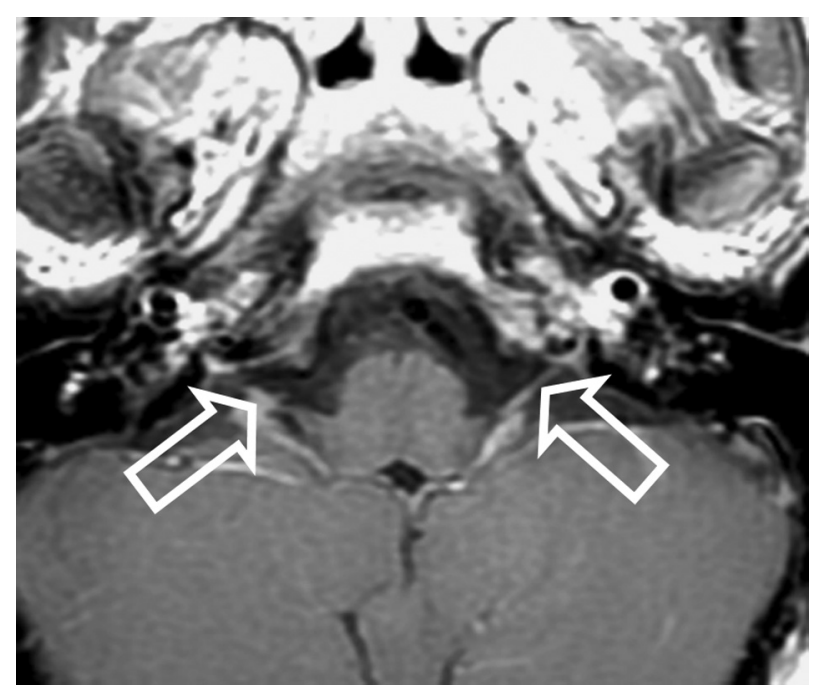

Fig 3. Patient 4. Bilateral enhancement of the eleventh cranial nerve is noted on an axial T1-weighted image (open arrows). 


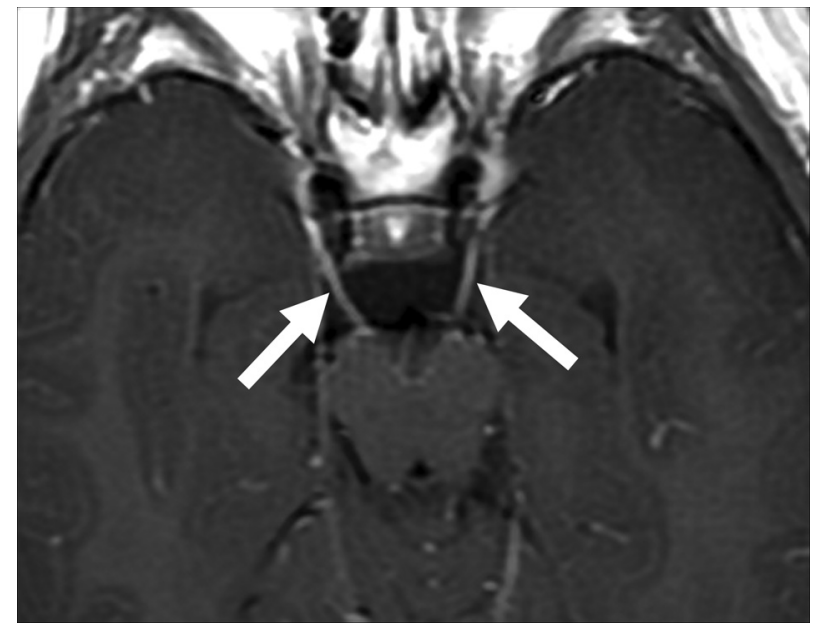

Fig 4. Patient 9. Bilateral enhancement of the third cranial nerve is observed on an axial T1-weighted image (solid arrows).

ings support the concept that pediatric GBS and MFS are acute polyneuropathies that represent a continuum rather than distinct entities, due to the overlap in their clinical and neuroradiologic features. In children affected by GBS-MFS, involvement of the CE roots may be considered part of a more extensive autoimmune neuropathy, as demonstrated by enhancement of cranial nerves. Brain MR imaging should be considered in the routine evaluation of pediatric patients with GBS-MFS. Cranial imaging in GBS-MFS may allow early detection of disease in patients with inconclusive neurologic examination findings or in patients with inconclusive spine MR imaging findings. These observations also support the notion that cranial neuroimaging could become a more central part of the baseline assessment and treatment-monitoring plan and that cranial nerve enhancement may become part of clinical diagnostic criteria for GBS and MFS if these findings could be reproduced in a large prospective study of cranial nerve involvement in GBS and MFS. Our study is limited, due its retrospective and observational design. Also, our MR imaging

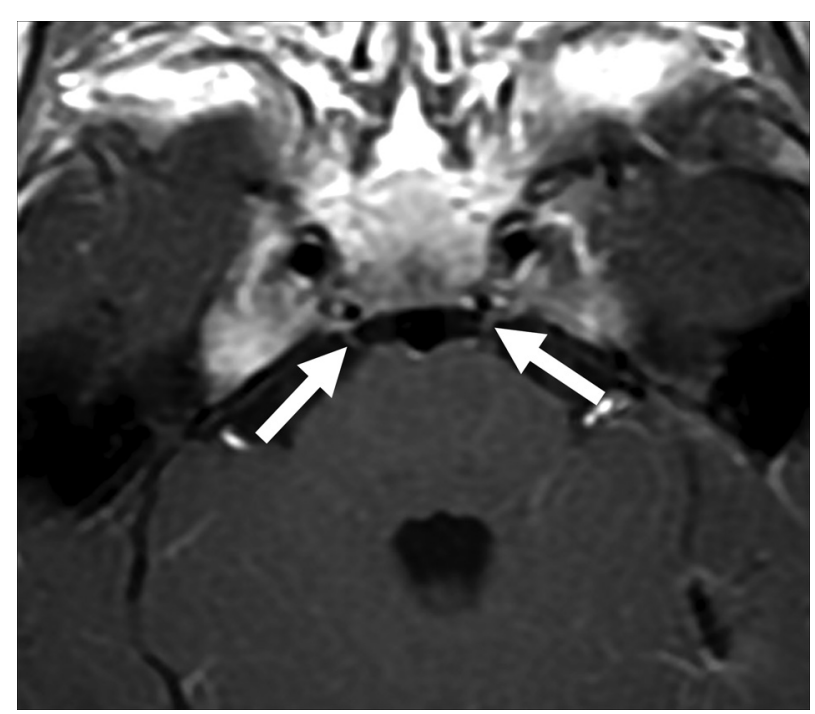

Fig 5. Patient 9. Bilateral enhancement of the sixth cranial nerve is seen on an axial T1-weighted image (solid arrows).

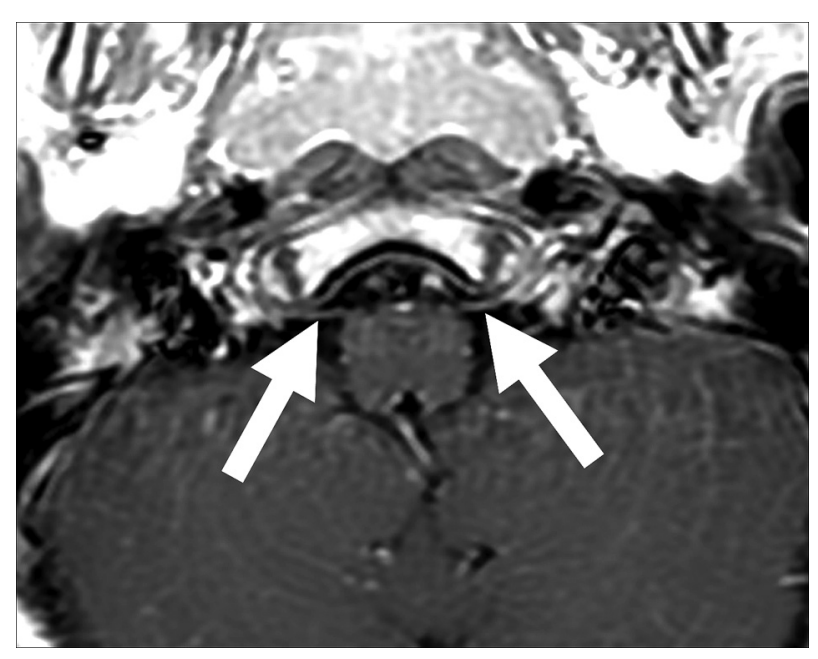

Fig 6. Patient 9. Bilateral subtle enhancement of the twelfth cranial nerve is seen on an axial T1-weighted image (solid arrows).

techniques were difficult to completely standardize during a 9 -year period.

\section{Involvement of the CE Roots}

Thickening and contrast enhancement in the CE roots on MR imaging is characteristic of patients with GBS and has been shown to be present in 95\% of typical patients with GBS. ${ }^{12-16}$ Although prominent nerve root enhancement has been observed along with an increased severity of leg weakness, ${ }^{14}$ the correlation between intracranial findings and clinical outcomes is poorly established. ${ }^{13,17,18}$ It was previously suggested that enhancement solely of the anterior CE roots was indicative of GBS, but it is now known that both the anterior and posterior roots may be simultaneously involved. ${ }^{13,18,19} \mathrm{We}$ found that the involvement of the anterior CE roots was more intense and frequent, thus confirming that though nonspecific, selective or relatively more intense enhancement of the anterior CE roots favors the diagnosis of GBS.

\section{Involvement of the Cranial Nerves}

Cranial involvement in pediatric patients with GBS has been reported in the literature, but the frequency and extent of involvement are unclear. ${ }^{20-23}$ A recent study of GBS in a pediatric population found that brain $\mathrm{MR}$ imaging findings of the patients were all within normal limits and did not show any abnormality concerning the cranial nerves. ${ }^{18}$ However, 5 of our patients had involvement of at least 1 cranial nerve (Figs $1-6)$.

GBS is clinically known to commonly involve the facial nerves, but most interesting, facial nerve enhancement has only been rarely reported on MR imaging. ${ }^{24}$ In this study, we did find contrast enhancement of the facial nerves in 1 patient, though 5 patients presented with facial nerve palsy. Furthermore, of the 3 patients who demonstrated involvement of the oculomotor cranial nerve, only 1 had ophthalmoplegia. The remainder of the involved cranial nerves as demonstrated by MR imaging had no clinical association, thus suggesting that enhancement does not necessarily correlate with the clinical symptoms. 


\section{Conclusions}

Even though MFS is represented by the classic triad of ophthalmoplegia, ataxia, and areflexia, MFS can present with only 1 of these features. ${ }^{11}$ If we accept this definition of MFS, then all of our patients met the diagnostic criteria for both GBS and MFS. Furthermore, we have shown that the neuroimaging features of GBS and MFS overlap. Our findings support the hypothesis that GBS and MFS represent the same clinical-neuroradiologic entity. The observation of a high incidence of cranial nerve enhancement in these pediatric patients with GBS-MFS suggests the possibility that routine cranial imaging could be integrated into the work-up of these patients.

\section{References}

1. Chio A, Cocito D, Leone M, et al. Guillain-Barré syndrome: a prospective, population-based incidence and outcome survey. Neurology 2003;60:1146-50

2. Hughes RA, Rees JH. Clinical and epidemiologic features of Guillain-Barré syndrome. J Infect Dis 1997;176:S92-98

3. Alter M. The epidemiology of Guillain-Barré syndrome. Ann Neurol 1990;27:S7-12

4. Alkan O, Yildirim T, Tokmak N, et al. Spinal MRI findings of Guillain-Barré syndrome. Neuroradiology 2009;3:25-28

5. Winer JB, Hughes RA, Anderson MJ, et al. A prospective study of acute idiopathic neuropathy. II. Antecedent events. J Neurol Neurosurg Psychiatry 1988;51:613-18

6. Burns TM. Guillain-Barré syndrome. Semin Neurol 2008;28:152-67

7. van Doorn PA, Ruts L, Jacobs BC. Clinical features, pathogenesis, and treatment of Guillain-Barré syndrome. Lancet Neurol 2008;7:939-50

8. Fulbright RK, Erdum E, Sze G, et al. Cranial nerve enhancement in the Guillain-Barré syndrome. AJNR Am J Neuroradiol 1995;16:923-25
9. Fisher M. An unusual variant of acute idiopathic polyneuritis (syndrome of ophthalmoplegia, ataxia and areflexia). N Engl J Med 1956;255:57-65

10. Collier J. Peripheral neuritis (Morison Lectures). Edinburgh Med J 1932;39: 601-18

11. Snyder LA, Rismondo V, Miller NR. The Fisher variant of Guillain-Barré syndrome (Fisher syndrome). J Neuroophthalmol 2009;29:312-24

12. Baran GA, Sowell MK, Sharp GB, et al. MR findings in a child with GuillainBarré syndrome. AJR Am J Roentgenol 1993;161:161-63

13. Coskun A, Kumandas S, Pac A, et al. Childhood Guillain-Barré syndrome: MR imaging in diagnosis and follow-up. Acta Radiol 2003;44:230-35

14. Iwata F, Utsumi Y. MR imaging in Guillain-Barré syndrome. Pediatr Radiol 1997;27:36-38

15. Gorson KC, Ropper AH, Muriello MA, et al. Prospective evaluation of MRI lumbosacral nerve root enhancement in acute Guillain-Barré syndrome. Neurology 1996;47:813-17

16. Matsuki M, Okada N, Matsuo M. Case report: MR findings of Guillain-Barré syndrome. Radiat Med 1998;16:133-35

17. Seneviratne U. Guillain-Barré syndrome. Postgrad Med J 2000;76:774-82

18. Yikilmaz A, Doganay S, Gumus H, et al. Magnetic resonance imaging of childhood Guillain-Barré syndrome. Childs Nerv Syst 2010;26:1103-08

19. Byun WM, Park WK, Park BH, et al. Guillain-Barré syndrome: MR imaging findings of the spine in eight patients. Radiology 1998;208:137-41

20. Armit R, Shapira Y, Blank A, et al. Acute, severe, central and peripheral nervous system combined demyelination. Pediatr Neurol 1986;2:47-50

21. Gamstrop I. Encephalo-myelo-radiculo-neuropathy: involvement of the CNS in children with Guillain-Barré-Strohl syndrome. Dev Med Child Neurol 1974; $16: 654-58$

22. Green SH. Polyradiculitis (Landry-Guillain-Barré syndrome) with total external ophthalmoplegia: encephalo-myelo-radiculo-neuropathy. Dev Med Child Neruol 1976;18:369-73

23. Okumura A, Ushida H, Maruyama K, et al. Guillain-Barré syndrome associated with central nervous system lesions. Arch Dis Child 2002;86:304-06

24. Yardimci N, Avci AY, Kayhan E, et al. Bilateral facial nerve enhancement demonstrated by magnetic resonance imaging in Guillain-Barré syndrome. $\mathrm{Neu}$ rol Sci 2009;30:431-33 